\title{
Visual field map clusters in human cortex
}

\author{
Brian A. Wandell ${ }^{1,2,3, *}$, Alyssa A. Brewer ${ }^{2}$ and Robert F. Dougherty ${ }^{3}$ \\ ${ }^{1}$ Psychology Department, ${ }^{2}$ Neurosciences Program, Psychology Department, and ${ }^{3}$ Stanford Institute for \\ Reading and Learning, Stanford University, fordan Hall, Building 420, Stanford, CA 94305-2130, USA
}

\begin{abstract}
We describe the location and general properties of nine human visual field maps. The cortical location of each map, as well as many examples of the eccentricity and angular representations within these maps, are shown in a series of images that summarize a large set of functional MRI data. The organization and properties of these maps are compared and contrasted with descriptions by other investigators. We hypothesize that the human visual field maps are arranged in several clusters, each comprising a group of maps that share a common foveal representation and semicircular eccentricity map. The spatial organization of these clusters suggests that the perceptual processing within each cluster serves related functions.
\end{abstract}

Keywords: visual cortex; retinotopy; fMRI; visual map clusters

\section{INTRODUCTION}

Neural signal processing takes place across an enormous range of scales. Molecular computations within the synapse take place at a sub-micron range; sensory computations may require comparisons between synaptic signals separated by hundreds of microns; and cortical structures may communicate information over a distance of many centimetres. The need to organize signals ranging from the molecular to those on the scale of the full brain places extraordinary demands on the brain structure. The nature of these demands can be seen in the startling observation that the axons in a single cubic millimetre of cortex extend $3-4 \mathrm{~km}$ in length (Braitenberg \& Schüz 1998).

Cortical maps are an important architectural method for organizing this information (Mountcastle 1957; Kaas 1997a). Each cortical map facilitates the comparison and combination of the information carried by various specialized neuronal populations. For example, visual field maps preserve the spatial structure of the scene itself: nearby scene points are represented in the responses of nearby neurons. Visual field maps then facilitate computations involving spatially localized comparisons between neurons specialized for carrying information about colour, motion or orientation.

Perhaps the best understood visual field map architecture is that of the retina. The cone mosaics in the retina sample the image and form three interleaved visual field maps; each map measures a different spectral band. Subsequent retinal wiring compares and combines signals from these cone mosaics into a dozen different retinal ganglion cell mosaics with receptive fields that measure different spatial, chromatic and temporal image features of the image. These output neurons again comprise a set of mosaics that are

* Author for correspondence (wandell@stanford.edu).

One contribution of 12 to a Theme Issue 'Cerebral cartography 1905-2005'. arranged as interleaved visual field maps. The basic architecture of the retina then begins with the cone mosaics that comprise interleaved visual field maps. These mosaics measure different aspects of the signal. The interleaved maps form the basis of retinal computations that compare nearby values in the image. The output is again an interleaved array of retinal ganglion cell mosaics arranged as maps.

The spatial coordination of signals at the neuronal scale may be paralleled by a spatial coordination at a coarser level. At a scale of several centimetres, we find that human maps seem to be arranged in spatial clusters. The spatial organization of the maps themselves may also play a role in coordinating neural computations. We suspect that within each cluster neurons share common computational resources. For example, the neurons might share short-term information storage or mechanisms that coordinate neural timing (Wandell et al. 2002). It may be that functional specializations for perception are organized around the activities within these clusters rather than single visual field maps (Bartels \& Zeki 2000).

On this view, information is organized at several scales. The interleaved neural mosaics within a visual field map permit dense interconnections between units that process very similar information. Clusters of visual field maps, in turn, coordinate information sharing between maps while minimizing long-range axonal connections (Kaas 1997b). We hypothesize that the functions carried out in clusters represent common perceptual processes, while the functions in different clusters are relatively independent, much as audition and vision are loosely coordinated.

The ability to measure visual field maps in the human brain, using functional magnetic resonance imaging (fMRI), offers an exciting opportunity to locate the maps, see their overall spatial arrangement and clarify their computational functions. In the main portion of this review, we summarize our understanding of the position and organization of human visual field maps. Several laboratories have been actively engaged in 

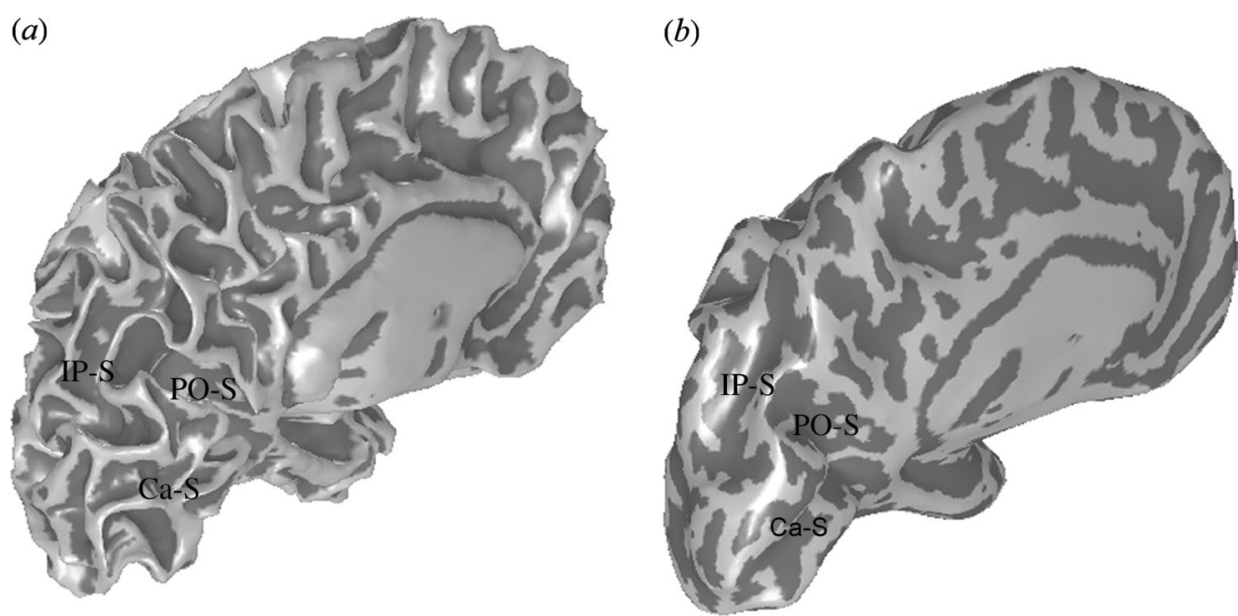

Figure 1. Rendering the cortical surface. (a) A rendering of a white-grey matter boundary in the human brain. The surface is estimated from anatomical MRI scans $\left(1 \times 1 \times 1 \mathrm{~mm}^{3}\right)$. The shading indicates the local curvature: light shading indicates a gyrus and dark shading a sulcus. (b) The same surface is rendered after smoothing. The shading on the smoothed surface is the same as in the original. In the following figures, we illustrate the properties and positions of the visual field maps on the smooth surface to reveal more of the data in a single image while still providing the general cortical position. Ca-S: calcarine sulcus. POS: parietal-occipital sulcus. IP-S: intraparietal sulcus.

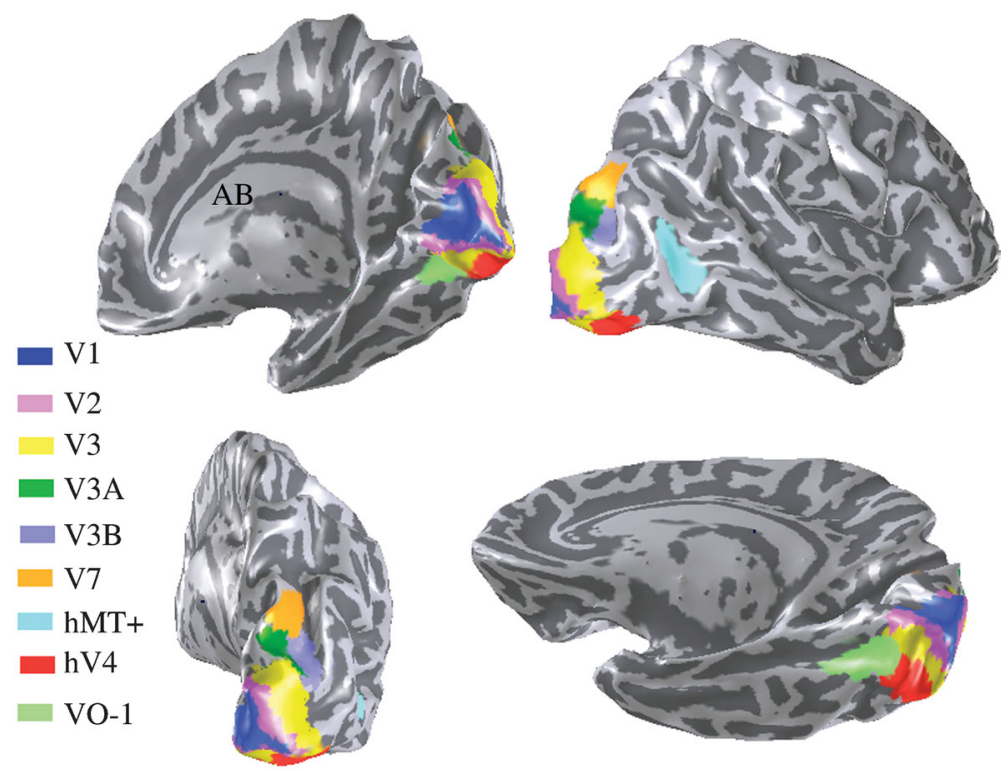

Figure 2. The locations of nine hemifield maps in the human visual cortex. The maps are shown for one typical subject (AB).

making these measurements and there are many areas of agreement. But there are also several specific differences, and we take this opportunity to explain vigorously our position on these differences.

\section{OVERVIEW}

To evaluate experimental work on visual field maps requires some familiarity with the methods used to represent the data. The field has made good progress in simplifying and clarifying the data presentation (Goebel 1998; Dale et al. 1999; Fischl et al. 1999; Wandell et al. 2000b), but several aspects of the methods are not widely understood. To simplify the presentation in this review, we use a single intuitive format for the images. In this section, we describe how we represent the surface of the brain and the visual field map data.

\section{(a) Surface representations}

We illustrate the positions and properties of visual field maps on reconstructions of the surface boundary between brain white matter and grey matter (figure 1). This boundary is identified using anatomical MR scans that have good contrast between grey and white matter. Automated segmentation algorithms provide the initial estimate of the boundary and an experienced investigator then verifies and edits these estimates (Teo et al. 1997; Wandell et al. 2000b). Once the boundary is identified, a triangular mesh of the surface is created. This surface mesh is used to visualize the location of the data on the surface.

The many folds in the human brain occlude much of the surface from view (figure 1a). To make more of the surface visible from a single viewpoint, we display this surface as a smoothed three-dimensional representation of the white-grey matter boundary 
(figure $1 b$ ). Smoothing exposes more of the surface in a single view while still retaining a general sense of the location of the main landmarks. The surface shading indicates whether each region of cortex was originally located in a sulcus (dark) or gyrus (light) on the original surface. The software used to produce these images is available from the authors.

\section{(b) Measuring maps}

Human fMRI methods for measuring visual field maps, called either travelling-wave or phase-encoded methods, are described in many publications (Engel et al. 1994, 1997; Sereno et al. 1995; DeYoe et al. 1996; Wandell 1999). Here, we offer a brief guide to the decisions investigators make when measuring visual field maps. These comments are intended to help the reader evaluate the data and interpretation of the maps.

The travelling-wave method measures the angular directions and the eccentricity bands that excite various cortical locations. For example, to measure eccentricity, a set of contrast patterns is presented in an orderly sequence from fovea to periphery. The eccentricity and angular directions of the stimulus that most effectively drives each cortical location is estimated from the pattern of responses.

Obtaining a high quality map requires choosing a set of travelling-wave stimuli that is appropriate to the cortical region under study. The stimulus set range and sampling density both influence the precision of the measurement. Suppose a region of cortex maps the central 3 degrees of vision precisely; using a sequence of stimuli outside of the map range, say positioned at 4,8 and 16 degrees, will fail to reveal the map. If the range of the stimulus set is improved to 2, 4 and 8 degrees, the response pattern will produce some information. However, the precision of the measurements will be poor because the estimated map is derived by interpolation of the responses of a very small number of effective samples.

Some investigators focus their measurements on a small number of spatial samples, such as two angular values (horizontal and vertical meridians; see Hasson et al. 2002; Fize et al. 2003) and two to four eccentricity bands (Hadjikhani et al. 1998; Hasson et al. 2003). When using coarsely sampled stimuli, many cortical locations are weakly driven because no stimulus falls in their preferred visual field location. Consequently, the fMRI response at those cortical locations is determined mainly by signals that spread from nearby cortex. The estimated stimulus preference then depends on the spatial spread (blurring) of the fMRI signal. The blurring process may depend on aspects of the brain that do not reflect the neural response at that cortical location.

A thorough quantitative simulation relating receptive field properties, the spreading of the vascular signal and measurement noise would be welcome; however, we are aware of only a few quantitative treatments of this important topic (Smith et al. 2001; Baseler et al. 2002; Logothetis \& Wandell 2004). Still, with regard to visual field mapping, certain general principles are clear. First, there is no strict connection between linear receptive field size and the presence of a map. A region of cortex may contain neurons with very small linear receptive fields; however, if the receptive fields are not
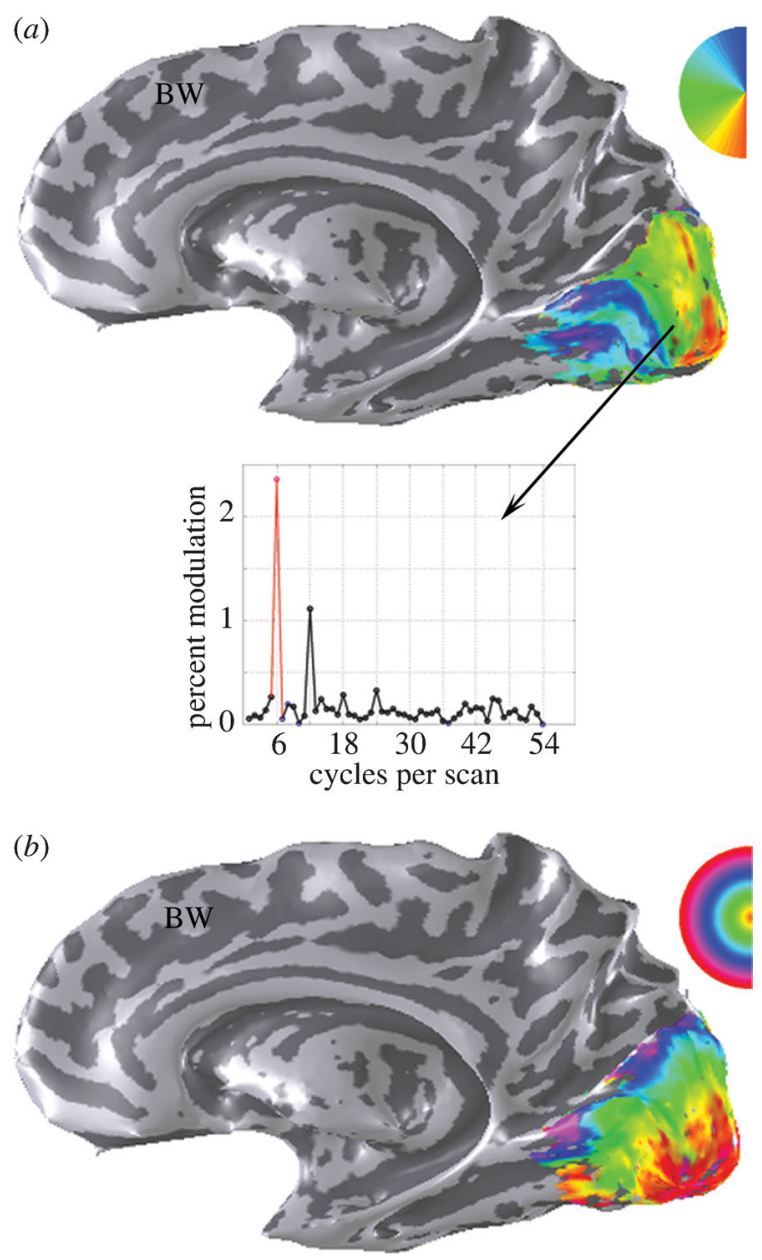

Figure 3. Angular and eccentricity maps near the calcarine cortex. Maps were measured using (a) rotating wedges and (b) expanding rings comprising contrast-reversing dartboard patterns (Wandell 1999). The stimuli extended over the central 20 degrees of the visual field and completed six cycles during each experimental scan. The colour overlay indicates the visual field angle $(a)$ or eccentricity $(b)$ that produces the most powerful response at each cortical location (see the coloured legends on the right). For clarity, only responses near the calcarine cortex are shown. The graph plots the response amplitude as a function of temporal frequency as measured in a $3 \mathrm{~mm}$ radius disk located in the calcarine (see arrow). The response is significantly greater at the stimulus repetition frequency ( 6 cycles per scan, shown in red) than other temporal frequencies. The secondary peaks at integer multiples of the stimulus frequency are expected and are also significant. Throughout, we include these graphs in images to provide the reader with an assessment of the reliability of the responses. The stimulus-driven responses shown in this paper are substantially above statistical threshold $(p<0.001$, uncorrected).

organized into a map, then the travelling-wave experiments will not produce a significant signal. On the other hand, regions of cortex may contain neurons with very large receptive fields that are organized into visual maps. More averaging is required to measure such maps because neurons with large receptive fields, by definition, do not respond much more to one position than another. This does not imply that the map is not present; it only implies that measuring maps in cortical regions containing large receptive fields requires a better signal. 
Second, the failure to find a map does not have the same significance as the discovery of a map. There are many technical reasons why one might fail to find a cortical map, so the inability to find a map with a few stimuli does not imply a map would not be found later. On the other hand, when a reliable map is found across multiple observers, the results should be accepted and form the basis for further study.

\section{(c) Visual field map positions}

The positions of nine visual hemifield field maps are shown in figure 2. These maps are present in both hemispheres; each map represents the contralateral visual field. In humans, the positions and general properties of the three visual field maps are agreed upon by all investigators: V1, V2 and V3. The V1 map falls mainly in the calcarine sulcus though it frequently extends beyond the posterior pole onto the ventral-lateral surface. The V2 and V3 maps form cortical strips that surround V1.

There is also agreement on the existence of a human V3A map on the dorsal surface at the posterior section of the intraparietal sulcus. An additional map, V3B, abuts V3A and has been described by two groups (Smith et al. 1998; Press et al. 2001). This small hemifield map shares a foveal representation with V3A and extends generally in the lateral direction. Two groups have identified a third hemifield map, V7, located anterior to V3A and V3B (Tootell et al. 1998; Press et al. 2001). The V7 foveal representation is distinct and anterior to that of V3A and V3B.

Our group has identified two ventral surface visual maps that represent the full contralateral hemifield (Wade et al. 2002; Brewer et al. submitted). These maps extend into the temporal lobe. One map (hV4) abuts the ventral V3 representation. The second (VO-1) is anterior and adjacent to hV4.

There is good agreement on the presence of a region of motion selectivity on the lateral surface of the occipital lobe near the temporal lobe (Zeki et al. 1991; Tootell et al. 1995; Wandell 1999; Zeki 2004). This region, hMT + , includes at least one map (Huk et al. 2002). The ' + ' symbol is appended to the name because, in addition to the human homologue of macaque MT (V5), hMT + is likely to contain additional cortical maps.

Sereno et al. (2001) identified a distinct visual map in the posterior parietal cortex that they describe as associated with eye movements and saccades. We remark briefly on the location and activity in this region because we measure activation there without eye movements and saccades. Our data are too preliminary to confidently label this region as a visual field map or as Sereno's posterior parietal map. A topographic area in macaque, V6 (PO), has been identified (Colby et al. 1988; Galletti et al. 1999) and there is a proposed human homologue of it in the parietal-occipital sulcus (Jousmaki et al. 1996). Because the topography of this region is not established in humans, this topic is not further reviewed (Portin \& Hari 1999; Dechent \& Frahm 2003).

The size, visual field representations, and functional signals of these visual field maps differ considerably. Also, the perceptual consequences of damage in the regions of each of these maps differ. In the following, we offer specific descriptions about the position and properties of these maps, and we relate our discussion to other descriptions in the literature. This paper represents a progress report, not a final report. We have no doubt that additional maps are present.

\section{POSTERIOR-MEDIAL MAPS (V1, V2, V3)}

The posterior-medial surface, extending from the occipital pole anterior along the calcarine sulcus, contains three hemifield maps. The primary visual cortex (V1), which receives direct input from the retino-geniculate pathway, occupies the calcarine cortex. Two additional maps (V2, V3) occupy a strip of cortex, roughly $1-3 \mathrm{~cm}$ wide, which encircles V1. Within these maps, the cortex responds powerfully to many visual stimuli. Localized cortical damage typically results in a general loss of visual function restricted to a corresponding region within the visual field (Horton \& Hoyt 1991a,b). Although damage causes severe visual dysfunction, in some neurological cases residual visual capabilities remain (Cowey 2004; Weiskrantz 2004).

The V1/V2/V3 hemifield maps can be identified from fMRI measurements of the eccentric and angular representations (figure 3; Engel et al. 1994, 1997a; Sereno et al. 1995; DeYoe et al. 1996). Measurements of the eccentricity representation, using an expanding ring stimulus, produce a single, large, continuous eccentricity map (figure $3 b$ ). Central visual field stimuli are represented on the ventral-lateral surface near the occipital pole; peripheral field stimuli are represented at increasingly anterior positions forming a semicircular pattern.

The angular measurements divide this unified eccentricity representation into several distinct maps. Combining the eccentricity and angular representations, we find a continuous hemifield map in calcarine (V1), as well as two additional maps, V2 and V3, that comprise two long strips of cortex surrounding V1. Each of these strips contains a discontinuous hemifield map, which is divided along the horizontal meridian. This discontinuity has the effect of producing maps with one long edge representing the horizontal meridian and a second representing the vertical meridian. The organization of these three visual field maps is much the same in humans and macaques.

These three maps are arranged in a configuration that co-registers several features of the maps. Figure $3 b$ shows clearly that the eccentricity representations of these maps are in register. In addition, vertical meridian representations of $\mathrm{V} 1 / \mathrm{V} 2$ are adjacent to one another, as are the horizontal meridian representations of V2/V3. Perhaps the only way to bring the various maps into closer alignment would be to do away with the distinct maps altogether and merge the cell mosaics in these three maps into a single map. Given the large number of mosaics known to exist in V1, incorporating additional mosaics may exceed the required incremental wiring within grey matter.

The cortical surface area representing the central visual field far exceeds that devoted to the peripheral field (Inouye 1909; Talbot \& Marshall 1941; Daniel \& 
Whitteridge 1961). Several groups have reported measurements of the absolute size and relative magnification of these human maps using fMRI (Horton \& Hoyt 1991b; Dougherty et al. 2003; Ejima et al. 2003). The surface area of human V1 and V2 varies by more than a factor of two between individuals, and the surface area of the primary visual cortex is strongly correlated with the size of the lateral geniculate nucleus and optic nerve (Andrews et al. 1997). There is also a large difference in the cone density across observers (Curcio et al. 1987, 1990). While the cone density and V1 surface area have not yet been measured in the same individual, this should be possible using adaptive optics. Hence, it is possible to test the hypothesis that the source of the V1 area variation begins with the determination of cone density (Roorda \& Williams 1999; Artal et al. 2004).

\section{(a) Related work}

As Brodmann emphasized in his remarkable book, the general properties of the layers and cells in the primary visual cortex (V1) are common across many species, including monkeys and humans (Brodmann 1909; Garey 1999). In these two species, the V2 map is roughly $80 \%$ of the surface area of the V1 map (Brewer et al. 2002; Dougherty et al. 2003). In addition, the spatial arrangement of human V1 and V2 maps matches that in the macaque visual cortex. However, significant quantitative differences exist. The macaque V1 has only half the surface area $\left(2000 \mathrm{~mm}^{2}\right.$; Brewer et al. 2002) of the human V1 (4000 $\mathrm{mm}^{2}$; Dougherty et al. 2003). Such a clear difference in size has not been reported in optic nerve or lateral geniculate size, leaving open the possibility that there are significant differences in the number of cortical neurons per optic nerve fibre.

Comparisons between human and macaque V3 maps suggest some quantitative differences, although these comparisons are complicated by the fact that estimates of the relative size of macaque V3 differ considerably. Lyon \& Kaas (2002) describe measurements of the macaque $\mathrm{V} 3$ as spanning roughly $5-8 \mathrm{~mm}$ at different eccentricities. Previous anatomical estimates proposed that the V3 strip is only $2-4 \mathrm{~mm}$ wide (Van Essen et al. 1986; Gattass et al. 1988). Using fMRI in macaque, Brewer et al. (2002) measured the width along the surface of the folded cortex and found it to be $9 \mathrm{~mm}$, comparable to that measured by Lyon and Kaas. Fize et al. (2003) replicated most of the results in Brewer et al., and specifically describe a V3 map. Measuring on the flattened representation, their strip appears $3 \mathrm{~mm}$ wide (see their figs. 1 and 8). However, estimates from flat maps are unreliable because of their distortions, and the identification of two boundaries separated by $3 \mathrm{~mm}$ is improbably small given that they used a voxel size of $2 \mathrm{~mm}$ followed by spatial smoothing. Hence, although they describe their data as consistent with that of Gattass, we conclude that their data follow those of Brewer and are consistent with the larger estimates of Lyon and Kaas.

\section{VENTRAL MAPS (hV4, VO-1)}

The ventral surface, extending from occipital into temporal cortex, is presently the subject of intense investigation. This region of cortex responds powerfully and selectively during object recognition tasks as subjects view a series of targets including faces, objects, text or even simple coloured patterns (Kanwisher et al. 1997; Epstein \& Kanwisher 1998; Cohen et al. 2000; Ishai et al. 2000). Damage in this region can result in face blindness, colour dysfunction or alexia (Meadows 1974a,b; Damasio et al. 1980, 1982; Michel et al. 1986, 1989; Sakurai 2004).

Our group has documented two hemifield maps in the ventral cortex, summarized in figure 4 (Wade et al. 2002; Brewer et al. submitted). One map directly abuts the ventral portion of $\mathrm{V} 3$ and shares a common eccentricity orientation with $\mathrm{V} 1 / \mathrm{V} 2 / \mathrm{V} 3$. We refer to this map as $\mathrm{hV} 4$ because part of this map has already been named V4 by other investigators. We add the ' $h$ ' to clarify that this map may not be homologous to V4 in other species. The hV4 eccentricity representation parallels that of $\mathrm{V} 1 / \mathrm{V} 2 / \mathrm{V} 3$, although the map may differ quantitatively (Ejima et al. 2003).

There is a second ventral hemifield map, VO-1, anterior to $\mathrm{hV} 4$. The data from the 3 degree expanding ring and rotating wedge experiments are shown in figure 4 . The posterior portion of VO-1 is adjacent to the relatively peripheral visual field representation of $\mathrm{hV} 4$; the VO-1 map also abuts the peripheral $\mathrm{V} 3 \mathrm{v}$ representation on the lingual gyrus (the peripheral V3 border is not shown for these 3 degree data). The posterior border of VO-1 represents the lower vertical meridian (red/yellow) and the anterior region represents the upper vertical meridian (cyan/blue). In this subject, the angular map runs in the anterior-ventral to medial direction; the direction of map is variable across observers compared with other maps, such as V1.

The VO-1 map extends into the temporal lobe, does not share a common eccentricity map with hV4 and has a distinct anterior foveal representation. We refer to this second ventral hemifield map as VO-1 because it is found in the ventral-occipital (VO) cortex, and we wish to allow for the possibility that additional maps (VO-2, VO-3, etc.) may be identified in the future.

There is a large emphasis on the foveal representation on the ventral surface; stimuli in the central 5 degrees evoke powerful responses within this region and there are much weaker responses to stimuli in the peripheral visual field. Hence, the ventral occipital maps are clarified by using stimuli with a range and sampling density that emphasize the central 5 degrees of the visual field.

\section{(a) Related work}

There is a significant dispute concerning the organization of ventral maps. The dispute includes two types of measurements: visual field maps and responses to colour stimuli. The summary of the maps provided above is based on our measurements; we do not mean to suggest that there is a consensus. Our understanding of the differences and an explanation of our view follow.

Zeki and his colleagues described a region of the ventral cortex that responds powerfully as one introduces colour into a monochrome pattern (Lueck et al. 1989; Zeki et al. 1991). McKeefry \& Zeki (1997) 
(a)
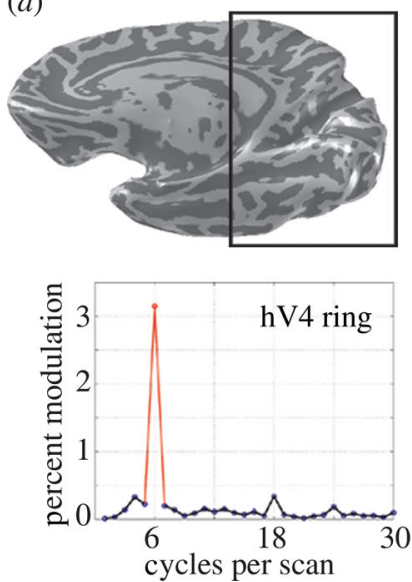

(b)

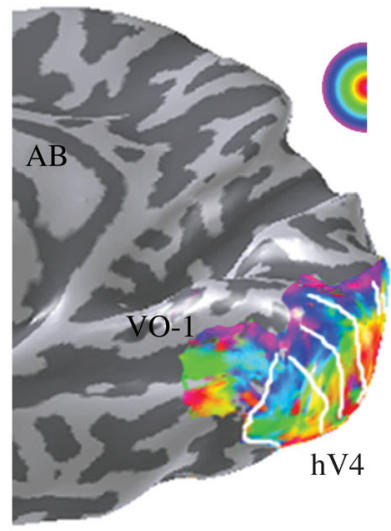

(c)

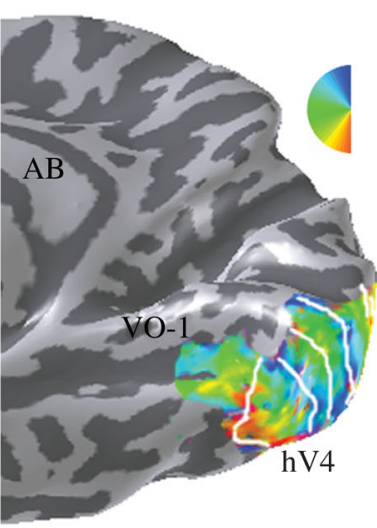

Figure 4. Eccentricity and angular measurements illustrating ventral occipital maps hV4 and VO-1. (a) The right hemisphere ventral occipital region of interest. (b) Eccentricity measurements using a 3 degree expanding ring stimulus. The graph shows the signal amplitude measured in a $5 \mathrm{~mm}$ radius disk centred in hV4. The response to the wedge in VO- 1 was of comparable magnitude. (c) Angle measurements using a 3 degree rotating wedge stimulus. White lines indicate the estimated boundaries between several visual field maps, including the ventral portions of V1, V2 and V3 (maps not labelled) as well as hV4 and VO (labelled). Other details as in figure 3.

further showed that the posterior part of this region is retinotopically organized and represents an entire hemifield. They did not measure the other visual field maps near this region, such as V2 and V3, leaving open how their map is positioned with respect to these other maps.

Tootell's group described two ventral visual field maps (Hadjikhani et al. 1998). The first is an upper quarterfield map, which they refer to as $\mathrm{V} 4 \mathrm{v}$, that abuts the central visual field representation of $\mathrm{V} 3 \mathrm{v}$ with an eccentricity map paralleling V1/V2/V3. This is one half of the map we identify as hV4. They found no adjacent lower quarterfield map. Instead, they describe a hemifield map with an eccentricity representation that runs perpendicular to the V4v quarter field, (fovea-periphery running lateral-medial). They named this map V8.

However, what of the lower quarterfield representation that one might expect to find associated with their putative V4v? Following the model of a macaque cortex, Tootell \& Hadjikhani (2001) searched for this quarterfield map in the dorsal cortex. They failed to find the map and concluded that it does not exist. We differ from this conclusion: specifically, we measure a lower quarterfield representation on the ventral surface adjacent to the upper quarterfield map they label as $\mathrm{V} 4 \mathrm{v}$ (Wade et al. 2002; Brewer et al. submitted).

We have considered the possibility that the only difference between the V4v/V8 model and our data is that Hadjikhani et al. (1998) missed this lower quarterfield in $\mathrm{hV} 4$. In this case, one might argue that $\mathrm{hV} 4$ and V8 coexist and that VO- 1 is really the same as V8. This predicts a modified V8 visual field map with (i) its peripheral representation abutting the hV4 lower vertical meridian and (ii) an angular representation, which runs parallel to the hV4 eccentricity map. As we explain in detail elsewhere, this model does not fit the VO-1 data (Brewer et al. submitted).

The data we present are consistent with data from Kastner et al. (2001) who describe a hemifield adjacent to $\mathrm{V} 3 \mathrm{v}$ and find no evidence for the V8 visual field map.
Oddly, Tootell's group subsequently published data consistent with a full visual hemifield map adjacent to V3v (Sasaki et al. 2001, fig. 5; Tootell \& Hadjikhani 2001, figs. 5 and 6). The organization of the ventral maps was secondary in those papers, and the authors did not discuss this aspect of their measurements. The presence of a full hemifield representation abutting V3 coupled with the VO-1 map obviates the proposed V4v/V8 model, although further study may justify a modification based on the quarter-field insertion concept.

While there is agreement that the ventral occipital cortex is essential for normal colour perception, the localization of colour processing with respect to individual maps is in dispute (Hadjikhani et al. 1998; Zeki et al. 1998; Zeki \& Bartels 1999; Bartels \& Zeki 2000). Zeki and his colleagues describe a V4-complex, whose position generally overlaps hV4 and the VO cluster, as the essential region for colour perception. Tootell and his colleagues propose that V8 is essential. While both laboratories identify essential colour signals by measuring the response to alternating stimulus pairs, the stimulus pairs are not comparable. The Zeki laboratory uses a pattern of rectangles and modulated chromatic contrast while maintaining luminance contrast constant. The Tootell laboratory uses harmonic patterns and alternates between a 95\% luminance and isoluminant stimuli; for this alternation both luminance and chrominance vary. These two types of stimulus exchanges should not produce identical cortical responses. For example, the Zeki stimuli would produce no modulation in pure luminance areas while the Tootell stimulus would.

As we explain elsewhere, these methods are not sufficiently precise to characterize the properties of cortical colour signals (Wade et al. 2002). A variety of factors, such as the spatial inhomogeneity of the retinal encoding of signals and better control of stimulus contrast, are essential for clarifying the colour signals present in the ventral surface (e.g. Engel et al. 1997b; Wandell et al. 2000a; Wade et al. 2002; Wade \& Wandell 2002). 
(a)

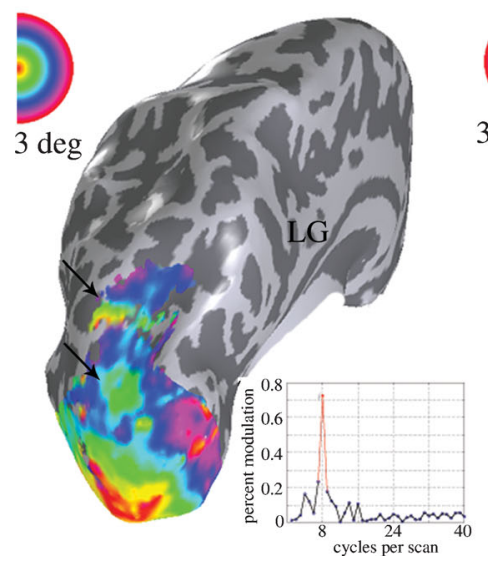

(b)

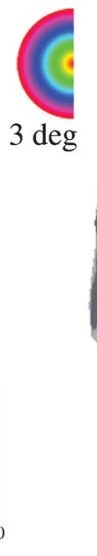

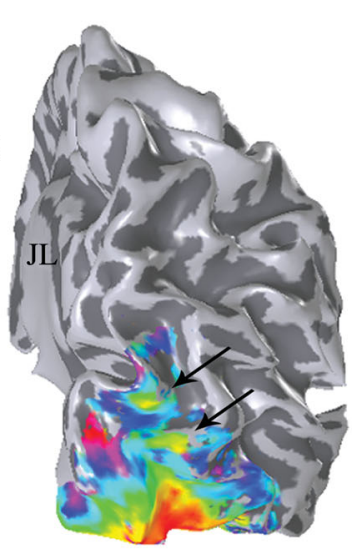

(c)

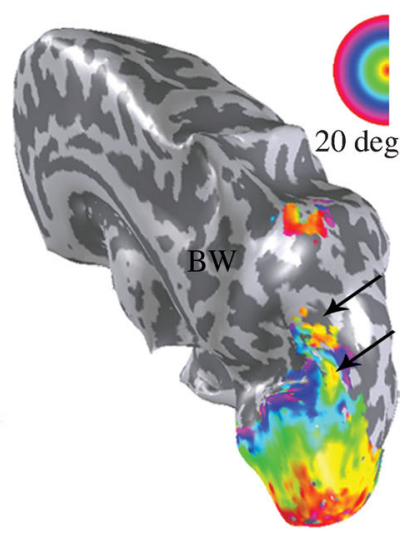

Figure 5. Eccentricity measurements in dorsal occipital maps V3A, V3B and V7. $(a, b)$ Eccentricity measurements using a 3 degree expanding ring stimulus in two subjects (LG and JL). The arrows indicate two foveal representations. The confluent foveal (yellow/green) representations of V3A and V3B can be seen in the relatively posterior region of the intraparietal sulcus. The graph measures the signal amplitude in this foveal representation (LG, $5 \mathrm{~mm}$ radius disk). The relatively anterior foveal representation is part of the V7 map. A fairly complete map is visible for LG and a partial but consistent map is seen in JL. (c) Eccentricity measurements using a 20 degree expanding ring (BW). The same eccentricity map is present, in particular note the semicircular map near V3A and V3B. In addition, note the foveal responses (orange/red) in the very anterior portion of the intraparietal sulcus. Such activity is frequently present and suggests that additional maps exist along the intraparietal sulcus (Sereno et al. 2001). For clarity, only responses near the intraparietal sulcus are shown. Other details as in figure 3.
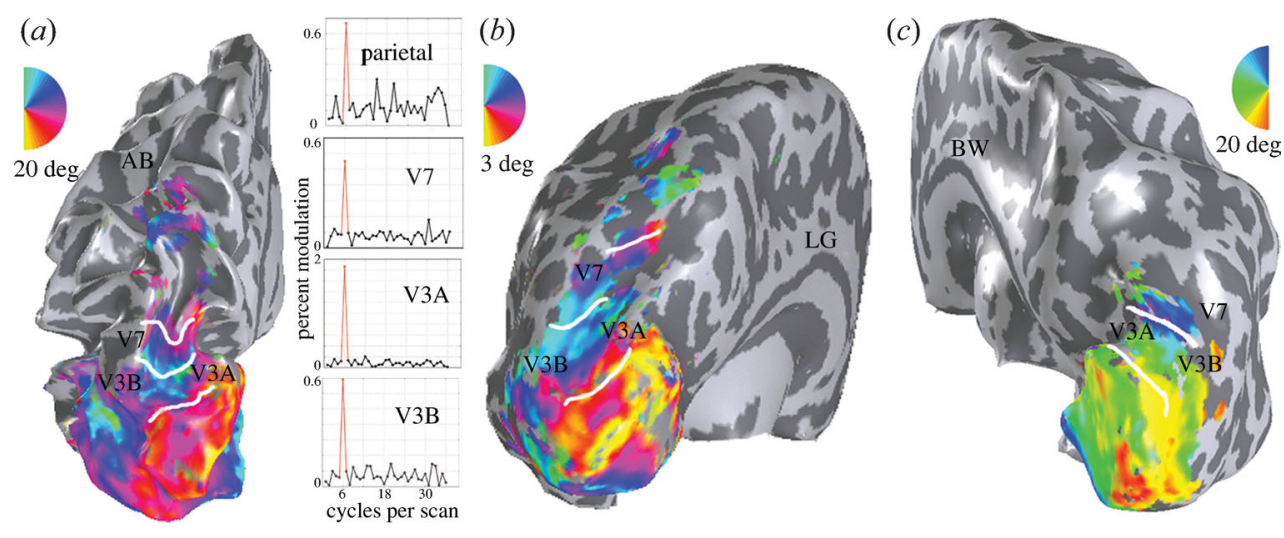

Figure 6. Angular measurements in dorsal occipital maps V3A, V3B and V7. $(a, b)$ Angular maps for V3A and V3B in the left hemisphere of LG and $A B$ were measured using 3 degree (LG) and 20 degree (AB) stimuli. The white lines delimit two hemifield maps, responding to lower (red/yellow) and upper (blue/cyan) vertical meridians. A lower vertical meridian representation separates $\mathrm{V} 3$ from $\mathrm{V} 3 \mathrm{~A} / \mathrm{V} 3 \mathrm{~B}$; an upper vertical meridian representation separates $\mathrm{V} 3 \mathrm{~A} / \mathrm{V} 3 \mathrm{~B}$ from $\mathrm{V} 7$; and a lower vertical meridian is present at the anterior boundary of V7. The graphs measure the relative reliability of the signals in the different maps as well as in the anterior intraparietal sulcus (AB). Note also the signals present in the anterior intraparietal sulcus. (c) Angular measurements in the right hemisphere using a 20 degree stimulus (BW). The lower (red/yellow) and upper (cyan/blue) vertical meridians, defining V3A and V3B, are clearly visible. The signal is weak at the anterior border of V7. The corresponding eccentricity maps for LG and BW are shown in figure 5. Corresponding measurements exist (not shown) for $\mathrm{AB}$ and JL. Other details as in figure 5.

\section{DORSAL MAPS (V3A, V3B, V7)}

The dorsal surface, extending from the posterior portion of the intraparietal sulcus forward, contains several small maps $\left(400-700 \mathrm{~mm}^{2}\right)$. Tasks involving motion and depth perception produce powerful responses in this region (Tootell et al. 1997; Paradis et al. 2000; Backus et al. 2001). Damage in this region can result in deficits when interpreting local motion signals (Vaina et al. 1998, 2003).

Three visual field maps (V3A, V3B and V7) can be measured in this region. Eccentricity maps from three subjects, representative of more than a dozen such maps, are shown in figure 5 . The confluence of V1/V2/ $\mathrm{V} 3$ forms a large foveal representation on the lateral aspect of the occipital pole. Two smaller foveal representations, separated from the V1/V2/V3 confluent foveal representation, are reliably found on the dorsal surface in the intraparietal sulcus (Press et al. 2001). In figure 5, the data from LG and JL were measured using expanding ring stimuli that swept out the central 3 degrees of the visual field. The measurements from $\mathrm{BW}$ were made using rings that extended to 20 degrees. The foveal representations have a natural variation in sulcal position comparable to that of other eccentricity maps (e.g. Dougherty et al. 2003).

To define the hemifield map requires coordinating eccentricity and angular maps; two representative angular maps are shown in figure 6 (see also Press et al. 
2001; Dumoulin et al. 2003). These maps overlay the two dorsal foveal representations, one angular map overlaying the posterior and the other overlaying the anterior foveal representation. Measuring from the posterior to the anterior cortex, the angular representations cover the lower to upper to lower vertical meridians.

To understand how the eccentricity and angular measurements define three hemifield maps consider the LG data. The eccentricity map near the foveal representation in the posterior intraparietal sulcus represents increasingly peripheral locations in the visual field in both lateral and medial directions. The corresponding angular map spans both the lateral and medial eccentricity representations. Hence, these measurements define two hemifield maps that share a confluent fovea. The relatively medial map is V3A; the relatively lateral map is $\mathrm{V} 3 \mathrm{~B}$. Just as visual maps $\mathrm{V} 1 / \mathrm{V} 2 / \mathrm{V} 3$ share a common foveal region with a semicircular eccentricity representation that emanates outward, so do V3A and V3B.

The anterior eccentricity representation runs lateralmedial (fovea-periphery) and the angular representation runs posterior-anterior (upper-lower). This defines a third hemifield map, V7 (Tootell et al. 1998; Press et al. 2001). We believe that further signal processing or improvements in techniques will identify additional maps within the intraparietal sulcus.

\section{(a) Related work}

A human V3A map was discussed in early cortical mapping papers (DeYoe et al. 1996); however, the first substantive study of the human V3A was from Tootell et al. (1997). The V3B map was suggested by Smith et al. (1998) and confirmed and clarified by others (Press et al. 2001; Dumoulin et al. 2003). Tootell et al. (1998) established the presence of at least a quarterfield V7 map; the existence of the map was confirmed and shown to represent hemifield by Press et al. (2001).

These intraparietal maps are difficult to measure because (i) the surface areas of the V3A, V3B and V7 maps $\left(400-700 \mathrm{~mm}^{2}\right)$ are smaller than that of the V1 map $\left(4000 \mathrm{~mm}^{2}\right)$ and (ii) signals in the intraparietal sulcus produced by simple rings and wedges are weaker than those in V1.

The literature concerning visual field maps near V3/V3A contains errors and inconsistencies. Tootell et al. (2003) describe only two hemifield maps, V3A and $\mathrm{V} 7$, and they incorrectly assert that $\mathrm{V} 3 \mathrm{~B}$ and $\mathrm{V} 7$ are the same. In recent reports, they do not describe the distinct foveal representation of V3A and V3B, although they were the first to suggest its presence (Tootell et al. 1997). The review by Orban et al. (2004) echoes this description by omitting V3B and incorrectly placing the human $\mathrm{V} 3 \mathrm{~A}$ foveal representation as confluent with that of $\mathrm{V} 1 / \mathrm{V} 2 / \mathrm{V} 3$.

Finally, we note an additional confusion about the responses in this general region. In an earlier analysis, Orban and his colleagues proposed the name $\mathrm{KO}$ to describe a cortical region particularly responsive to kinetic boundaries (Dupont et al. 1997; Van Oostende et al. 1997). They did not locate this region with respect to the retinotopic maps. Zeki et al. (2003) showed that $\mathrm{KO}$ was not uniquely responsive to kinetic boundaries as had been claimed. They speculated that the location of $\mathrm{KO}$ might be consistent with that of V3B. In an abstract and recent talks, Tyler et al. (2003) confirm Zeki's criticism - and that $\mathrm{KO}$ should be renamed. However, Tyler \& Wade (personal communication) measured retinotopic maps and locate the region lateral to V3B.

\section{LATERAL MAPS (hMT + , LOC)}

The lateral surface, extending from the occipital pole to the superior temporal sulcus, responds heterogeneously to visual stimuli. The anterior portion of the lateral occipital (LO) lobe, extending into the superior temporal sulcus, responds powerfully to motion and attention (Zeki et al. 1991; Tootell \& Taylor 1995; Tootell et al. 1997; Culham et al. 1998; Brefczynski \& DeYoe 1999). In one neurological case, extensive damage that includes this region caused a deficit in many, but not all, aspects of motion perception (Zihl et al. 1983; Shipp et al. 1994; Rizzo et al. 1995). Disruption of electrical signalling in this region of cortex, using transcranial magnetic stimulation or intracranial electrical stimulation, interferes specifically with motion perception (Hotson et al. 1994; Beckers \& Zeki 1995). Investigators commonly describe the posterior portion as the LO complex. In this region, the cortex responds powerfully during certain object recognition tasks (Malach et al. 1995; Grill-Spector et al. 1998). The surface area of the LO complex is commensurate with that of $\mathrm{V} 1$.

Huk et al. (2002; see also Dukelow et al. 2001) reported the existence of coarse maps within the motion responsive region $\mathrm{hMT}+$. The small size of the hMT + cortical map and the highly folded nature of the cortex make this region difficult to study. Nevertheless, since the original reports, we have been able to improve the quality of the measurements, and several examples of maps in hMT + are illustrated in figures 7 and 8 . While the presence of at least one map is certain, there may be multiple small maps in this region.

Our group routinely observes retinotopically organized responses on the lateral surface between V3 and $\mathrm{hMT}+$, overlapping with the LO complex. As the data in figures 7 and 8 illustrate, there are both eccentricity and angular maps in this region. In our early experimental measurements on the ventral surface we also occasionally saw maps. As we optimized and adequately averaged the stimulus properties, these ventral maps became increasingly clear and certain. The LO maps are not yet visible in every scan, and the pattern of data reminds us of our preliminary studies of ventral maps. We believe that as we continue to investigate and optimize the stimuli and methods, we will be able to confidently identify the organization of the visual field maps in this LO region.

\section{(a) Related work}

Tootell et al. (2001) measured eccentricity and angular representations in the LO cortex. They focused their search for a quarterfield representation that might be the homologue of monkey $\mathrm{V} 4 \mathrm{~d}$, and reported being unable to measure retinotopic maps in this region. Instead, they suggested that this region has an 
eccentricity bias in which adjacent regions respond preferentially to central and preferentially peripheral stimuli. Tootell and colleagues refer to the region of dorsal cortex where they fail to find the lower quarterfield map either as LOC/LOP (meaning lateral occipital central and lateral occipital peripheral, subdivisions), or the V4d-topo. In subsequent papers, the region is proposed as a visual area (Tootell et al. 2003; Tsao et al. 2003). Malach and his colleagues also suggest that the ventral and lateral cortex regions have eccentricity bias, but no angular maps (Levy et al. 2001; Hasson et al. 2002, 2003). Reviews by Van Essen (2003) and Orban et al. (2004) echo this view without additional data. Contrary to this summary, we find responses to both expanding ring and rotating wedge stimuli in the cortex between V3d and hMT + (see figures 7 and 8 ) and on the ventral surface (figure 4).

The LO data in Tootell et al. (2001) bear little resemblance to the data here. For example, in their fig. 7A the authors describe a sudden transition from central peripheral representation. This plot shows a preferred eccentricity change of 8 to 10 degrees of visual angle over a $2.5 \mathrm{~mm}$ cortical distance with sample spacing of $400 \mu \mathrm{m}$. Yet the inplane fMRI spatial resolution for each subject was $3.1 \times 3.1 \mathrm{~mm}^{2}$ (slice thickness $3-4 \mathrm{~mm}$; p. 300). In any single subject, the entire transition would take place within a single voxel. Hence, we think the authors made an error in quantifying these data.

Malach's group suggests that it is possible to measure eccentricity biases in this region of cortex, but not angular maps (Levy et al. 2001). Figure 8 illustrates two of several subjects in which we have measured strong responses to rotating wedges. We suspect that with better methods, including more averaging, different stimulus parameters and detailed study, reliable visual field maps will be identified on the lateral surface. We propose that maps in this region should follow the naming convention LO-1, LO-2, and so forth.

\section{DISCUSSION}

\section{(a) Visual map clusters}

In the Introduction we suggested that visual field maps coordinate signals across multiple levels of spatial resolution. Specifically at fine scales, it is beneficial to arrange neurons so that synaptic values that must be compared, differenced or summed are in close proximity. The visual field map data prompt the question of whether there are principles at a coarse scale that organize the spatial arrangements of the maps and nearby cortex.

We suggest that there is a similar principle that applies to the organization of the visual maps. Specifically, the maps are arranged in clusters when the neural mosaics in these maps serve similar common computational goals. We speculate that these map clusters share resources such as circuitry that coordinates the timing of neural signals or temporarily stores local calculations (short-term memory; Wandell et al. 2002).

This view contrasts with a suggestion by Malach and his colleagues (Levy et al. 2001; Hasson et al. 2002, 2003). They propose 'a new organizing principle in which object representations are arranged according to a central versus peripheral visual field bias' (Levy et al. 2001, abstract). According to this model, the visual cortex has a hierarchical organization that begins with the precise visual field maps in V1, V2 and V3. Retinotopic organization degrades, so that the lateral and ventral regions contain coarse eccentricity maps but 'the polar angle representation in these areas is cruder, and orderly representations of the visual field meridians are absent' (Levy et al. 2001, p. 533). As visual field maps degrade, they propose that the cortex is organized around representations of perceptual entities, such as faces, places, objects and the theoretical entities introduced by cognitive psychologists. Although these object-selective regions do not have a map organization, the positions of the object-selective regions align with the eccentricity maps. In particular, regions that respond best to foveated stimuli (letters, faces) are extensions of those portions of the early cortex that represent central vision; regions that respond best to large-scale representations (navigation, houses, places) align with those portions of the early cortex that represent peripheral vision. The model gives a prominent role to the eccentricity bias in that it might 'encompass essentially the entire extent of the human visual cortex' (Hasson et al. 2002, p. 479). This eccentricity bias is a unifying principle in that 'the entire set of object-related occipital-temporal regions could be placed, with minor distortions, into a single unified map of centre-periphery organization' (Hasson et al. 2003, p. 1028). An overview of this model can be found in Grill-Spector \& Malach (2004; p. 668 et seq. and fig. 12).

We find two problems with the eccentricity bias hypothesis, one theoretical and one empirical. First, no compelling computational principle has been identified to suggest why neurons would be arranged with an eccentricity, but no angular, bias. Second, we have not measured the regions of the cortex that have an eccentricity bias and no angular map. The data in figures 7 and 8 demonstrate detailed eccentricity and angular maps on the lateral and ventral surfaces. As the graphs in the figures illustrate, the responses to the travelling-wave stimuli are quite significant and this can only occur if the neurons in these cortical regions have a map-like organization. Because we frequently see eccentricity and angular maps in lateral and ventral occipital cortex, we explain a failure to find these maps as a limitation of the experimental methodology, not a property of the cortex.

Why do Malach and his colleagues find an eccentricity bias and no angular maps? Perhaps the differences can be explained by methodologies. Their studies were conducted using a $1.5 \mathrm{~T}$ magnet, while we used a $3 \mathrm{~T}$ magnet. They measured angular maps using only horizontal and vertical patterns, while we measured with 8 different orientations. And why did they see only an eccentricity bias rather than the detailed maps we describe? A further difference is that they measured with 3 eccentricity bands and left unstimulated gaps between these bands.

For these reasons, we propose an alternative model. The visual cortex appears to contain several distinct clusters of maps. Each cluster shares a confluent fovea 

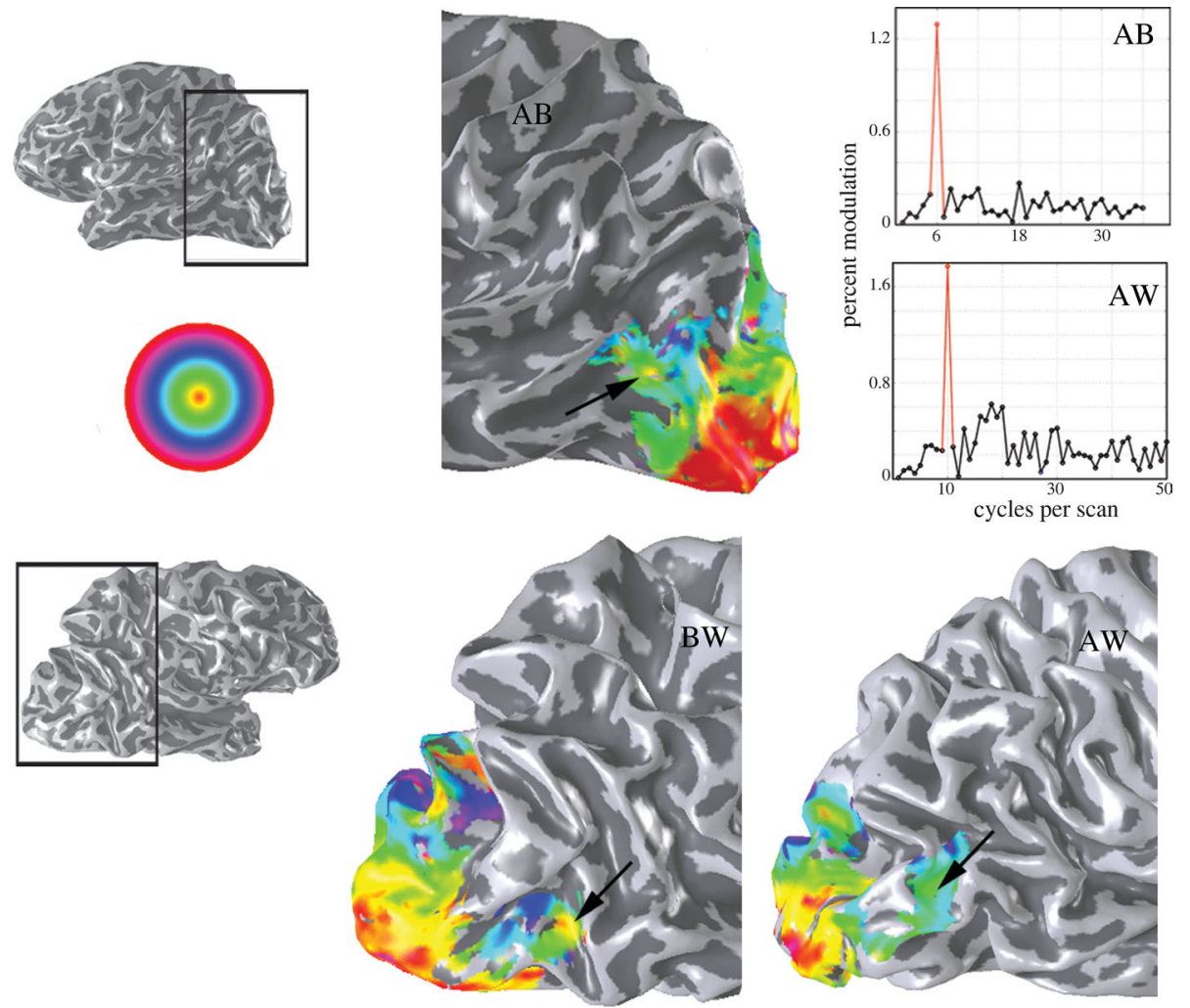

Figure 7. Eccentricity measurements in lateral occipital cortex. Regions of interest in the right and left lateral occipital cortex are shown in the images at the left. Eccentricity measurements using 20 degree expanding ring stimulus in one left hemisphere (AB) and two right hemispheres (BW, AW) are shown. The arrows indicate the semicircular eccentricity maps in hMT + . While the preferred central phase in $\mathrm{V} 1$ is fairly consistent (red), there is variation in the preferred central phase in hMT + , (orange, to yellow, to green). This variation colour is representative of our data. For clarity, only responses in the dorsal and lateral occipital cortex are shown. The graphs measure the signal amplitude in this region in two subjects ( $3 \mathrm{~mm}$ radius disk). Other details as in figure 3.

with semicircular eccentricity bands. The posterior cluster, centred on the occipital pole, is the most obvious. This cluster includes V1, V2, V3 and hV4. This semicircular map extends laterally, towards $\mathrm{hMT}+$; it remains to be seen how much of LO is an independent cluster and how much is part of the posterior cluster. This organization is repeated for the smaller distinct foveal representations, such as $\mathrm{V} 3 \mathrm{~A} / \mathrm{V} 3 \mathrm{~B}$ and $\mathrm{hMT}+$. We speculate that further measurements will identify new clusters near VO-1, V7 and the anterior portions of the intraparietal sulcus. The positions of these visual map clusters are indicated in figure 9.

\section{(b) The myth of the physiological gold standard}

Studies of human visual field mapping have been guided from the beginning by the extensive literature on the monkey visual cortex, and specifically macaque visual areas. This served as an excellent starting point for regions near the primary visual cortex. However, we question whether the macaque model of visual areas should continue to serve as a gold standard to guide analysis of human visual field maps (Zeki 2003).

In this chapter, we thought it important to describe measurements of 'visual field maps' and to avoid the phrase 'visual area'. We did this because the definition of a human visual field map is clear and closely coupled to a specific type of measurement. Visual area definitions, however, are based on a potentially conflicting set of criteria. As Van Essen writes, 'The identification of distinct visual areas is generally based on finding reliable differences in one or more characteristics related to: (i) architecture, (ii) connectivity, (iii) visual topography, and/or (iv) functional characteristics' (Van Essen 2003). According to this definition, any measured difference may be used to propose the presence of a visual area and the role of visual field maps (topography) can become secondary. This makes the connection between visual areas and visual field maps tenuous.

The history of the third visual field map in macaque, V3, exemplifies how the definition of visual field maps and visual areas can diverge. Well aware of the presence of a visual hemifield map, Van Essen and colleagues identified a variety of differences between the upper and lower field V3 representations. They proposed that these portions of the map be considered as different visual areas (V3 and VP; Burkhalter et al. 1986; Burkhalter \& Van Essen 1986). On this model, two putative visual areas, V3 and VP, form a single hemifield map. Following in this direction, Orban and colleagues are exploring the hypothesis that the macaque V4 map should be broken into two visual areas (Orban et al. 2004, p. 320-321). Perhaps the most extreme example is that of Tootell et al. (2001), who use the inability to measure a visual map predicted from macaque as a rationale to label a human visual area (V4d-topo).

A second reason for refraining from the use of visual areas in macaque to guide our understanding of human 


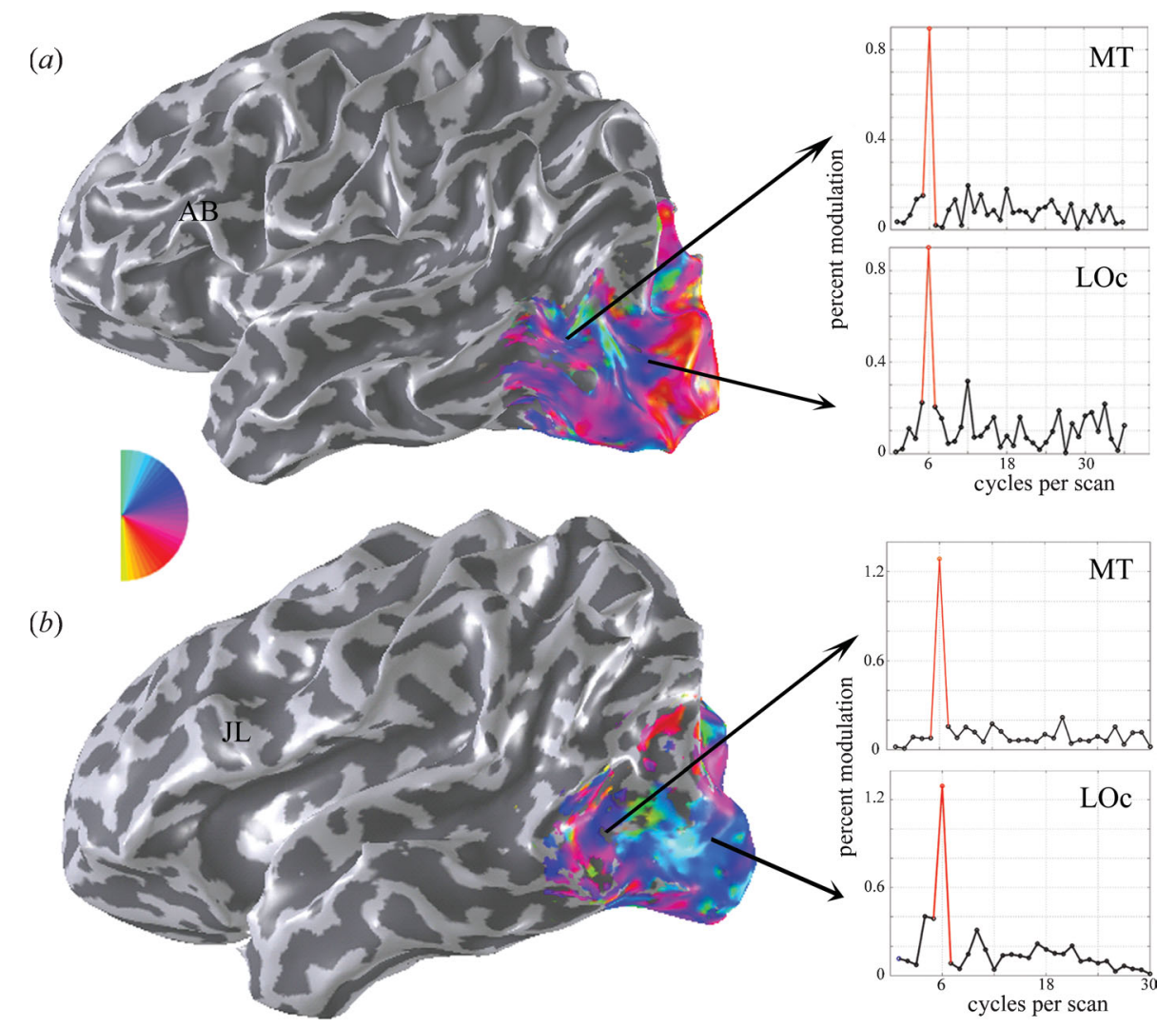

Figure 8. Angular measurements in the lateral occipital cortex. Rotating wedge measurements using 3 degree stimuli are shown for subject $\mathrm{AB}(a)$ and subject $\mathrm{JL}(b)$. The graphs indicate that there are significant responses to these stimuli in the lateral occipital cortex ( $3 \mathrm{~mm}$ radius disk). These data suggest that it will be possible to identify visual field maps. In this region, responses to 3 degree stimuli tend to be larger than responses to 20 degree stimuli (cf. figure 7 ). Other details as in figure 3.
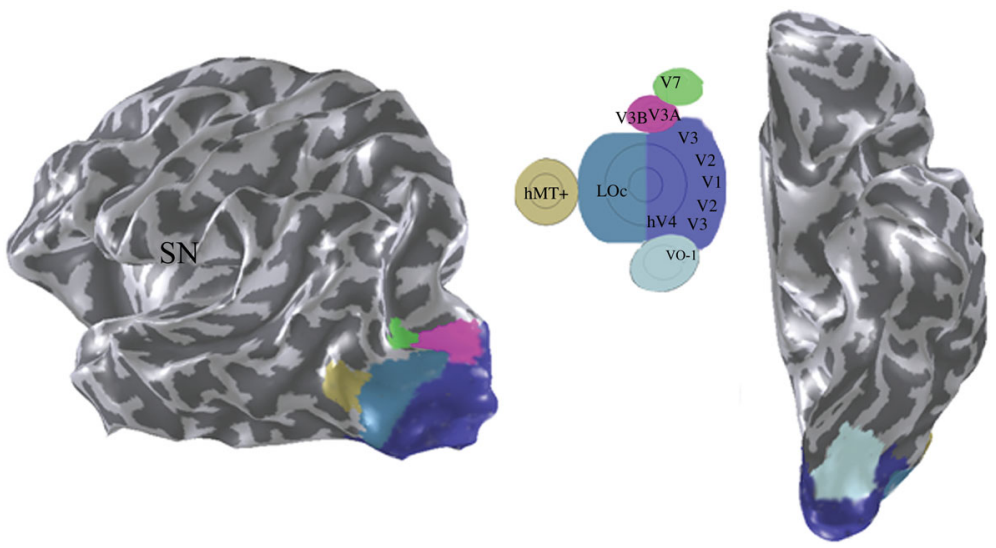

Figure 9. Six candidate visual map clusters. A suggested organization of the visual clusters in a typical subject (SN). Each cluster contains a foveal representation, semicircular eccentricity map and one or more hemifield maps. The images show a lateral (left) and ventral (right) view. The inset is a schematic flat map of the cluster organization. At least one map has been identified in five clusters, but others may be found. No maps have been securely identified in LOc, but the data in figures 7 and 8 suggest that maps will be found.

visual field maps flows from considerations of the relationship between maps and stimulus responsivity. Differences in stimulus responsivity across the map are considered as a reason to divide the map into distinct visual areas; however such a principle is problematic. For example, consider the midget ganglion cell visual field maps. There are many receptive field differences between foveal and peripheral midgets, both with respect to size, sampling density, colour tuning and so forth. Yet it would be unwise to divide this mosaic into different 'retinal areas'; similarly, it would be unwise to divide cortical areas, such as V1, into two areas based on the different stimulus responsivity in the fovea and periphery. As we develop an understanding of cortical organization, it seems important to separate the measurements of maps and the measurements of stimulus responsivity. The integration of measurements used to define visual areas creates significant complexity and confusion.

Another reason often given for using macaque visual areas to guide the interpretation of human visual field maps is based on a common misunderstanding of the 
current state of these fields. Although it comes as a surprise to many of our colleagues in psychology and neuroimaging, there is no consensus view on macaque visual areas (Van Essen 2003). Given the heroic nature of the methods required to obtain the macaque data, it will be some time before consensus is reached. The human literature should look to the physiological literature for guidance where there is consensus, as for V1, V2 and MT. However, beyond these visual areas, the limited consensus and enormous differences in the methodologies diminish the value of macaque visual areas as a gold standard for the human cortex.

Finally, efforts to integrate macaque visual areas with human visual field maps are strained because of the significant differences between the two species. We have noted several major differences, both quantitative and qualitative, in this review. These differences are not surprising given the 25 million years of evolutionary divergence (Hedges \& Kumar 2003) and may be more substantial than can be captured by map deformations (Van Essen et al. 2001). Visual map clusters and individual visual maps may exist in one species but not the other.

\section{CONCLUSION}

We described the organization of nine human visual hemifield maps that are present in each hemisphere. These maps were identified using a very limited number of experimental conditions. Expanding the range of stimuli and measurement conditions is likely to reveal additional maps, particularly in the LO cortex and along the intraparietal sulcus.

We introduced a specific hypothesis concerning the spatial organization of visual maps: we proposed that maps are organized into clusters. These clusters share a confluent fovea and a semicircular eccentricity map. This organizational principle is an alternative to the eccentricity bias theory recently proposed by Malach and collaborators, and the two theories have different implications for development and function. Rather than proposing a single unifying structure for all of the visual cortex, we suggest that there are several independent structures and that each serves a different perceptual computation. On this theory, one might expect that signals within map clusters are more thoroughly integrated than signals communicated between clusters. We might also expect that the maps within a cluster will have a similar developmental time course.

We are very hopeful that new neuroimaging techniques will permit tests of these hypotheses. For example, diffusion tensor imaging (DTI) provides a valuable measurement of brain structure. DTI coupled with fibre-tracing algorithms should become an important tool in determining how maps are connected (Conturo et al. 1999; Xue et al. 1999; Basser et al. 2000). Also, the spatial resolution of magnetic resonance spectroscopy is steadily increasing (Pfeuffer et al. 1999). The new high field (7 T) magnets may provide estimates of the chemical composition within different visual maps, providing insights about commonalities, differences and development.
As we imagine a future with many new methods, we can find inspiration in the pioneers who came before us. Brodmann, who we honour here, has influenced the work of generations by his experimental work and by establishing broad and ambitious goals. In summarizing his work, Brodmann wrote in very modern terms: 'my ultimate goal was the advancement of a theory of function and its pathological deviations' (Brodmann 1909 , p. 243). The enormous influence of that work is based on its breadth, precision and his choice to make measurements in the service of far-reaching goals (Brodmann 1909; Garey 1999). Modern tools will help us advance toward these goals if we strive to follow Brodmann's scientific standards and goals.

We thank Jonathan Horton, Semir Zeki, William Newsome, David Heeger, Kalanit Grill-Spector, Junjie Liu, Alex Wade, Joyce Farrell and Satoshi Nakadomari. Supported by NEI EY 03164 and NINDS 5F30 NS44759.

\section{REFERENCES}

Andrews, T. J., Halpern, S. D. \& Purves, D. 1997 Correlated size variations in human visual cortex, lateral geniculate nucleus, and optic tract. F. Neurosci. 17, 2859-2868.

Artal, P., Chen, L., Fernandez, E. J., Singer, B., Manzanera, S. \& Williams, D. R. 2004 Neural compensation for the eye's optical aberrations. F. Vis. 4, 281-287.

Backus, B. T., Fleet, D. J., Parker, A. J. \& Heeger, D. J. 2001 Human cortical activity correlates with stereoscopic depth perception. F. Neurophysiol. 86, 2054-2068.

Bartels, A. \& Zeki, S. 2000 The architecture of the colour centre in the human visual brain: new results and a review. Eur. F. Neurosci. 12, 172-193.

Baseler, H. A., Brewer, A. A., Sharpe, L. T., Morland, A. B., Jagle, H. \& Wandell, B. A. 2002 Reorganization of human cortical maps caused by inherited photoreceptor abnormalities. Nat. Neurosci. 5, 364-370.

Basser, P. J., Pajevic, S., Pierpaoli, C., Duda, J. \& Aldroubi, A. 2000 In vivo fiber tractography using DT-MRI data. Magn. Reson. Med. 44, 625-632.

Beckers, G. \& Zeki, S. 1995 The consequences of inactivating areas V1 and V5 on visual motion perception. Brain 118 (Pt 1), 49-60.

Braitenberg, V. \& Schüz, A. 1998 Cortex: statistics and geometry of neuronal connectivity, 2nd edn. Heidelberg, Germany: Springer-Verlag Telos.

Brefczynski, J. A. \& DeYoe, E. A. 1999 A physiological correlate of the 'spotlight' of visual attention. Nat. Neurosci. 2, 370-374.

Brewer, A. A., Press, W. A., Logothetis, N. K. \& Wandell, B. A. 2002 Visual areas in macaque cortex measured using functional magnetic resonance imaging. f. Neurosci. 22, $10416-10426$.

Brewer, A. A., Liu, J., Wade, A. R. \& Wandell, B. A. Submitted. Visual field maps and stimulus selectivity in human ventral occipital cortex. Nat. Neurosci.

Brodmann, K. 1909 Vergleichende lokalisationslehre der groSshirnrinde. Leipzig: Verlag von Johann Ambrosius Barth.

Burkhalter, A. \& Van Essen, D. C. 1986 Processing of color, form and disparity information in visual areas VP and V2 of ventral extrastriate cortex in the macaque monkey. f. Neurosci. 6, 2327-2351.

Burkhalter, A., Felleman, D. J., Newsome, W. T. \& Van Essen, D. C. 1986 Anatomical and physiological asymmetries related to visual areas V3 and VP in macaque extrastriate cortex. Vision Res. 26, 63-80.

Cohen, L., Dehaene, S., Naccache, L., Lehericy, S., Dehaene-Lambertz, G., Henaff, M. A. \& Michel, F. 
2000 The visual word form area: spatial and temporal characterization of an initial stage of reading in normal subjects and posterior split-brain patients. Brain 123 (Pt 2), 291-307.

Colby, C. L., Gattass, R., Olson, C. R. \& Gross, C. G. 1988 Topographical organization of cortical afferents to extrastriate visual area PO in the macaque: a dual tracer study. f. Comp. Neurol. 269, 392-413.

Conturo, T. E., Lori, N. F., Cull, T. S., Akbudak, E., Snyder, A. Z., Shimony, J. S., McKinstry, R. C., Burton, H. \& Raichle, M. E. 1999 Tracking neuronal fiber pathways in the living human brain. Proc. Natl Acad. Sci. USA 96, $10422-10427$.

Cowey, A. 2004 The 30th Sir Frederick Bartlett lecture. Fact, artefact, and myth about blindsight. Q. F. Exp. Psychol. A 57, 577-609.

Culham, J. C., Brandt, S. A., Cavanagh, P., Kanwisher, N. G., Dale, A. M. \& Tootell, R. B. 1998 Cortical fMRI activation produced by attentive tracking of moving targets. F. Neurophysiol. 80, 2657-2670.

Curcio, C. A., Sloan, Jr, K. R., Pakcer, O., Hendrickson, A. E. \& Kalina, R. E. 1987 Distribution of cones in human and monkey retina: individual variability and radial asymmetry. Science 236, 579-582.

Curcio, C. A., Sloan, K. R., Kalina, R. E. \& Hendrickson, A. E. 1990 Human photoreceptor topography. F. Comp. Neurol. 292, 497-523.

Dale, A. M., Fischl, B. \& Sereno, M. I. 1999 Cortical surfacebased analysis. I. Segmentation and surface reconstruction. Neuroimage 9, 179-194.

Damasio, A., Yamada, T., Damasio, H., Corbett, J. \& McKee, J. 1980 Central achromatopsia: behavioral, anatomic, and physiologic aspects. Neurology 30, 1064-1071.

Damasio, A. R., Damasio, H. \& Van Hoesen, G. W. 1982 Prosopagnosia: anatomic basis and behavioral mechanisms. Neurology 32, 331-341.

Daniel, P. M. \& Whitteridge, D. 1961 The representation of the visual field on the cerebral cortex in monkeys. F. Physiol. (Paris) 159, 203-221.

Dechent, P. \& Frahm, J. 2003 Characterization of the human visual V6 complex by functional magnetic resonance imaging. Eur. F. Neurosci. 17, 2201-2211.

DeYoe, E. A., Carman, G. J., Bandettini, P., Glickman, S., Wieser, J., Cox, R., Miller, D. \& Neitz, J. 1996 Mapping striate and extrastriate visual areas in human cerebral cortex. Proc. Natl Acad. Sci. USA 93, 2382-2386.

Dougherty, R. F., Koch, V. M., Brewer, A. A., Fischer, B., Modersitzki, J. \& Wandell, B. A. 2003 Visual field representations and locations of visual areas $\mathrm{V} 1 / 2 / 3$ in human visual cortex. F. Vis. 3, 586-598.

Dukelow, S. P., DeSouza, J. F., Culham, J. C., van den Berg, A. V., Menon, R. S. \& Vilis, T. 2001 Distinguishing subregions of the human $\mathrm{MT}+$ complex using visual fields and pursuit eye movements. F. Neurophysiol. 86, 1991-2000.

Dumoulin, S. O., Baker, C. L., Jr, Hess, R. F. \& Evans, A. C. 2003 Cortical specialization for processing first- and second-order motion. Cereb. Cortex 13, 1375-1385.

Dupont, P., De Bruyn, B., Vandenberghe, R., Rosier, A. M., Michiels, J., Marchal, G., Mortelmans, L. \& Orban, G. A. 1997 The kinetic occipital region in human visual cortex. Cereb. Cortex 7, 283-292.

Ejima, Y., Takahashi, S., Yamamoto, H., Fukunaga, M., Tanaka, C., Ebisu, T. \& Umeda, M. 2003 Interindividual and interspecies variations of the extrastriate visual cortex. Neuroreport 14, 1579-1583.

Engel, S. A., Rumelhart, D. E., Wandell, B. A., Lee, A. T., Glover, G. H., Chichilnisky, E. J. \& Shadlen, M. N. 1994
fMRI of human visual cortex [letter]. Nature 369, 525 [published erratum appears in Nature 1994 Jul 14;370(6485):106].

Engel, S. A., Glover, G. H. \& Wandell, B. A. $1997 a$ Retinotopic organization in human visual cortex and the spatial precision of functional MRI. Cereb. Cortex 7, 181-192.

Engel, S., Zhang, X. \& Wandell, B. $1997 b$ Colour tuning in human visual cortex measured with functional magnetic resonance imaging. Nature 388, 68-71.

Epstein, R. \& Kanwisher, N. 1998 A cortical representation of the local visual environment. Nature 392, 598-601.

Fischl, B., Sereno, M. I. \& Dale, A. M. 1999 Cortical surfacebased analysis. II. Inflation, flattening, and a surface-based coordinate system. Neuroimage 9, 195-207.

Fize, D., Vanduffel, W., Nelissen, K., Denys, K., Chef d'Hotel, C., Faugeras, O. \& Orban, G. A. 2003 The retinotopic organization of primate dorsal V4 and surrounding areas: a functional magnetic resonance imaging study in awake monkeys. $\mathcal{F}$. Neurosci. 23, 7395-7406.

Galletti, C., Fattori, P., Gamberini, M. \& Kutz, D. F. 1999 The cortical visual area V6: brain location and visual topography. Eur. F. Neurosci. 11, 3922-3936.

Garey, L. J., 1999 Brodmann's 'localisation in the cerebral cortex', London: Imperial College Press.

Gattass, R., Sousa, A. P. \& Gross, C. G. 1988 Visuotopic organization and extent of $\mathrm{V} 3$ and $\mathrm{V} 4$ of the macaque. F. Neurosci. 8, 1831-1845.

Goebel, R. 1998 Brainvoyager. Maastricht, The Netherlands: Brain Innovation.

Grill-Spector, K. \& Malach, R. 2004 The human visual cortex. Annu. Rev. Neurosci. 27, 649-677.

Grill-Spector, K., Kushnir, T., Edelman, S., Itzchak, Y. \& Malach, R. 1998 Cue-invariant activation in objectrelated areas of the human occipital lobe. Neuron 21, 191-202.

Hadjikhani, N., Liu, A. K., Dale, A. M., Cavanagh, P. \& Tootell, R. B. H. 1998 Retinotopy and color sensitivity in human visual cortical area V8. Nat. Neurosci. 1, 235-241.

Hasson, U., Levy, I., Behrmann, M., Hendler, T. \& Malach, R. 2002 Eccentricity bias as an organizing principle for human high-order object areas. Neuron 34, 479-490.

Hasson, U., Harel, M., Levy, I. \& Malach, R. 2003 Largescale mirror-symmetry organization of human occipitotemporal object areas. Neuron 37, 1027-1041.

Hedges, S. B. \& Kumar, S. 2003 Genomic clocks and evolutionary timescales. Trends Genet. 19, 200-206.

Horton, J. C. \& Hoyt, W. F. 1991 a Quadrantic visual field defects: a hallmark of lesions in extrastriate (V2/V3) cortex. Brain 114, 1703-1718.

Horton, J. C. \& Hoyt, W. F. $1991 b$ The representation of the visual field in human striate cortex. A revision of the classic Holmes map. Arch. Ophthalmol. 109, 816-824.

Hotson, J., Braun, D., Herzberg, W. \& Boman, D. 1994 Transcranial magnetic stimulation of extrastriate cortex degrades human motion direction discrimination. Vision Res. 34, 2115-2123.

Huk, A. C., Dougherty, R. F. \& Heeger, D. J. 2002 Retinotopy and functional subdivision of human areas MT and MST. F. Neurosci. 22, 7195-7205.

Inouye, T. 1909 Die sehstroungen bei schussverietzungen der kortikalen sehsphare. Leipzig, Germany: W. Engelmann.

Ishai, A., Ungerleider, L. G., Martin, A. \& Haxby, J. V. 2000 The representation of objects in the human occipital and temporal cortex. F. Cogn. Neurosci. 12, 35-51.

Jousmaki, V., Hamalainen, M. \& Hari, R. 1996 Magnetic source imaging during a visually guided task. Neuroreport 7, 2961-2964. 
Kaas, J. H. 1997a Theories of visual cortex organisation in primates. In Cerebral cortex, vol. 12 (ed. K. J. Rockland, J. H. Kaas \& A. Peters) Extrastriate cortex in primates, vol. 12, pp. 91-125. New York: Plenum Press.

Kaas, J. H. $1997 b$ Topographic maps are fundamental to sensory processing. Brain Res. Bull. 44, 107-112.

Kanwisher, N., McDermott, J. \& Chun, M. M. 1997 The fusiform face area: a module in human extrastriate cortex specialized for face perception. f. Neurosci. 17, 4302-4311.

Kastner, S., De Weerd, P., Pinsk, M. A., Elizondo, M. I., Desimone, R. \& Ungerleider, L. G. 2001 Modulation of sensory suppression: implications for receptive field sizes in the human visual cortex. f. Neurophysiol. 86, 1398-1411.

Levy, I., Hasson, U., Avidan, G., Hendler, T. \& Malach, R. 2001 Center-periphery organization of human object areas. Nat. Neurosci. 4, 533-539.

Logothetis, N. K. \& Wandell, B. A. 2004 Interpreting the BOLD signal. Annu. Rev. Physiol. 66, 735-769.

Lueck, C. J., Zeki, S., Friston, K. J., Deiber, M. P., Cope, P., Cunningham, V. J., Lammertsma, A. A., Kennard, C. \& Frackowiak, R. S. 1989 The colour centre in the cerebral cortex of man. Nature 340, 386-389.

Lyon, D. C. \& Kaas, J. H. 2002 Evidence for a modified V3 with dorsal and ventral halves in macaque monkeys. Neuron 33, 453-461.

Malach, R. et al. 1995 Object-related activity revealed by functional magnetic resonance imaging in human occipital cortex. Proc. Natl Acad. Sci. USA 92, 8135-8139.

McKeefry, D. J. \& Zeki, S. 1997 The position and topography of the human colour centre as revealed by functional magnetic resonance imaging. Brain 120, 2229-2242.

Meadows, J. C. $1974 a$ Disturbed perception of colours associated with localized cerebral lesions. Brain 97, 615-632.

Meadows, J. C. $1974 b$ The anatomical basis of prosopagnosia. F. Neurol. Neurosurg. Psychiatry 37, 489-501.

Michel, F., Perenin, M. T. \& Sieroff, E. 1986 Prosopagnosia without hemianopsia after unilateral right occipitotemporal lesion. Rev. Neurol. 142, 545-549.

Michel, F., Poncet, M. \& Signoret, J. L. 1989 Are the lesions responsible for prosopagnosia always bilateral? Rev. Neurol. 145, 764-770.

Mountcastle, V. B. 1957 Modality and topographic properties of single neurons of cat's somatic sensory cortex. f. Neurophysiol. 20, 408-434.

Orban, G. A., Van Essen, D. \& Vanduffel, W. 2004 Comparative mapping of higher visual areas in monkeys and humans. Trends Cogn. Sci. 8, 315-324.

Paradis, A. L., Cornilleau-Peres, V., Droulez, J., Van De Moortele, P. F., Lobel, E., Berthoz, A., Le Bihan, D. \& Poline, J. B. 2000 Visual perception of motion and 3-D structure from motion: an fMRI study. Cereb. Cortex 10, $772-783$.

Pfeuffer, J., Tkac, I., Provencher, S. W. \& Gruetter, R. 1999 Toward an in vivo neurochemical profile: quantification of 18 metabolites in short-echo-time (1)H NMR spectra of the rat brain. F. Magn. Reson. 141, 104-120.

Portin, K. \& Hari, R. 1999 Human parieto-occipital visual cortex: lack of retinotopy and foveal magnification. Proc. R. Soc. B 266, 981-985.

Press, W. A., Brewer, A. A., Dougherty, R. F., Wade, A. R. \& Wandell, B. A. 2001 Visual areas and spatial summation in human visual cortex. Vision Res. 41, 1321-1332.

Rizzo, M., Nawrot, M. \& Zihl, J. 1995 Motion and shape perception in cerebral akinetopsia. Brain 118 (Pt 5), 1105-1127.
Roorda, A. \& Williams, D. R. 1999 The arrangement of the three cone classes in the living human eye. Nature 397, 520-522.

Sakurai, Y. 2004 Varieties of alexia from fusiform, posterior inferior temporal and posterior occipital gyrus lesions. Behav. Neurol. 15, 35-50.

Sasaki, Y., Hadjikhani, N., Fischl, B., Liu, A. K., Marrett, S., Dale, A. M., Tootell, R. B. \& Marret, S. 2001 Local and global attention are mapped retinotopically in human occipital cortex. Proc. Natl Acad. Sci. USA 98, 2077-2082.

Sereno, M. I., Dale, A. M., Reppas, J. B., Kwong, K. K., Belliveau, J. W., Brady, T. J., Rosen, B. R. \& Tootell, R. B. 1995 Borders of multiple visual areas in humans revealed by functional magnetic resonance imaging. Science 268, 889-893.

Sereno, M. I., Pitzalis, S. \& Martinez, A. 2001 Mapping of contralateral space in retinotopic coordinates by a parietal cortical area in humans. Science 294, 1350-1354.

Shipp, S., de Jong, B. M., Zihl, J., Frackowiak, R. S. \& Zeki, S. 1994 The brain activity related to residual motion vision in a patient with bilateral lesions of V5. Brain 117 (Pt 5), 1023-1038.

Smith, A. T., Greenlee, M. W., Singh, K. D., Kraemer, F. M. \& Hennig, J. 1998 The processing of first- and second-order motion in human visual cortex assessed by functional magnetic resonance imaging (fMRI). F. Neurosci. 18, 3816-3830.

Smith, A. T., Singh, K. D., Williams, A. L. \& Greenlee, M. W. 2001 Estimating receptive field size from fMRI data in human striate and extrastriate visual cortex. Cereb. Cortex 11, 1182-1190.

Talbot, S. \& Marshall, W. 1941 Physiological studies on neural mechanisms of visual localization and discrimination. Am. F. Ophthalmol. 24, 1255-1263.

Teo, P. C., Sapiro, G. \& Wandell, B. A. 1997 Creating connected representations of cortical gray matter for functional MRI visualization. IEEE Trans. Med. Imaging 16, 852-863.

Tootell, R. B. \& Hadjikhani, N. 2001 Where is 'dorsal V4' in human visual cortex? Retinotopic, topographic and functional evidence. Cereb. Cortex 11, 298-311.

Tootell, R. B. \& Taylor, J. B. 1995 Anatomical evidence for $\mathrm{MT}$ and additional cortical visual areas in humans. Cereb. Cortex 1, 39-55.

Tootell, R. B., Reppas, J. B., Kwong, K. K., Malach, R., Born, R. T., Brady, T. J., Rosen, B. R. \& Belliveau, J. W. 1995 Functional analysis of human MT and related visual cortical areas using magnetic resonance imaging. F. Neurosci. 15, 3215-3230.

Tootell, R. B., Mendola, J. D., Hadjikhani, N. K., Ledden, P. J., Liu, A. K., Reppas, J. B., Sereno, M. I. \& Dale, A. M. 1997 Functional analysis of V3A and related areas in human visual cortex. F. Neurosci. 17, 7060-7078.

Tootell, R. B., Hadjikhani, N., Hall, E. K., Marrett, S., Vanduffel, W., Vaughan, J. T. \& Dale, A. M. 1998 The retinotopy of visual spatial attention. Neuron 21, 1409-1422.

Tootell, R. B., Tsao, D. \& Vanduffel, W. 2003 Neuroimaging weighs in: humans meet macaques in "primate" visual cortex. F. Neurosci. 23, 3981-3989.

Tsao, D. Y. et al. 2003 Stereopsis activates V3A and caudal intraparietal areas in macaques and humans. Neuron 39, 555-568.

Tyler, C. W., Likova, L. T., Wade, A. R. \& Kontsevich, L. L. 2003 Cortical area KO responds well to stereoscopic structure Abstract Viewer/Itinerary Planner, Program No. 339.11. Washington, DC: Society for Neuroscience. 
Vaina, L. M., Makris, N., Kennedy, D. \& Cowey, A. 1998 The selective impairment of the perception of first-order motion by unilateral cortical brain damage. Vis. Neurosci. 15, 333-348.

Vaina, L. M., Gryzwacz, N. M., Saiviroonporn, P., LeMay, M., Bienfang, D. C. \& Cowey, A. 2003 Can spatial and temporal motion integration compensate for deficits in local motion mechanisms? Neuropsychologia 41, 1817-1836.

Van Essen, D. C. 2003 Organization of visual areas in macaque and human cerebral cortex. In The visual neurosciences (eds. L. M. Chalupa \& J. S. Werner), pp. 507-521. Boston: Bradford Books.

Van Essen, D. C., Newsome, W. T., Maunsell, J. H. \& Bixby, J. L. 1986 The projections from striate cortex (V1) to areas V2 and V3 in the macaque monkey: asymmetries, areal boundaries, and patchy connections. F. Comp. Neurol. 244, 451-480.

Van Essen, D. C., Lewis, J. W., Drury, H. A., Hadjikhani, N., Tootell, R. B., Bakircioglu, M. \& Miller, M. I. 2001 Mapping visual cortex in monkeys and humans using surface-based atlases. Vision Res. 41, 1359-1378.

Van Oostende, S., Sunaert, S., Van Hecke, P., Marchal, G. \& Orban, G. A. 1997 The kinetic occipital (KO) region in man: an fMRI study. Cereb. Cortex 7, 690-701.

Wade, A. R. \& Wandell, B. A. 2002 Chromatic light adaptation measured using functional magnetic resonance imaging. f. Neurosci. 22, 8148-8157.

Wade, A. R., Brewer, A. A., Rieger, J. W. \& Wandell, B. A. 2002 Functional measurements of human ventral occipital cortex: retinotopy and colour. Phil. Trans. R. Soc. B 357, 963-973.

Wandell, B. A. 1999 Computational neuroimaging of human visual cortex. Annu. Rev. Neurosci. 22, 145-173.

Wandell, B., Baseler, H., Boynton, G. \& Engel, S. $2000 a$ Computational neuroimaging: color tuning in two human cortical areas measured using fMRI. In Color vision: from molecular genetics to perception (ed. K. Gegenfurtner \& L. T. Sharpe), Cambridge: Cambridge University Press.
Wandell, B. A., Chial, S. \& Backus, B. $2000 b$ Visualization and measurement of the cortical surface. F. Cogn. Neurosci. 12, 739-752.

Wandell, B. A., El Gamal, A. \& Girod, B. 2002 Common principles of image acquisition systems and biological vision. Proc. IEEE 90, 5-17.

Weiskrantz, L. 2004 Roots of blindsight. Prog. Brain Res. 144, 229-241.

Xue, R., van Zijl, P. C., Crain, B. J., Solaiyappan, M. \& Mori, S. 1999 In vivo three-dimensional reconstruction of rat brain axonal projections by diffusion tensor imaging. Magn. Reson. Med. 42, 1123-1127.

Zeki, S. 2003 Improbable areas in the visual brain. Trends Neurosci. 26, 23-26.

Zeki, S. 2004 Thirty years of a very special visual area, area V5. F. Physiol. 557, 1-2.

Zeki, S. \& Bartels, A. 1999 The clinical and functional measurement of cortical (in)activity in the visual brain, with special reference to the two subdivisions (V4 and V4 alpha) of the human colour centre. Phil. Trans. R. Soc. B 354, 1371-1382.

Zeki, S., Watson, J. D., Lueck, C. J., Friston, K. J., Kennard, C. \& Frackowiak, R. S. 1991 A direct demonstration of functional specialization in human visual cortex. f. Neurosci. 11, 641-649.

Zeki, S., McKeefry, D. J., Bartels, A. \& Frackowiak, R. S. 1998 Has a new color area been discovered? Nat. Neurosci. 1, 335-336.

Zeki, S., Perry, R. J. \& Bartels, A. 2003 The processing of kinetic contours in the brain. Cereb. Cortex 13, 189-202.

Zihl, J., von Cramon, D. \& Mai, N. 1983 Selective disturbance of movement vision after bilateral brain damage. Brain 106 (Pt 2), 313-340.

\section{GLOSSARY}

DTI: diffusion tensor imaging

fMRI: functional magnetic resonance imaging 